\title{
LA COMPOSICIÓN DE LA PERSONA EN EL PENSAMIENTO RARÁMURI
}

María Isabel Martínez Ramírez

IIA UNAM

\section{RESUMEN}

El objetivo de este artículo es identificar la composición de la persona rarámuri y describir cada uno de sus elementos. Más allá de reconocer la noción de cuerpo y de las entidades que lo animan, en el análisis se enfatizarán las relaciones que dan pie a nuevos componentes de tipo emocional, cognitivo y onírico. La salida del alma es un eje fundamental en el pensamiento rarámuri, a través de ella se explican los estados de saludenfermedad y vida-muerte. El presente es un texto etnográfico que busca contribuir al conocimiento antropológico de las culturas del norte de México, particularmente del noroeste.

Palabras clave: Ráramuri, persona, cuerpo, alma, 


\begin{abstract}
The proposal of this paper is to identify the composition of the raramuri person and, to describe each part of its components. Beyond recognizing the notion of the body and the entities that animate it, this investigation emphasizes those relations that allow new emotional, cognitive and oniric components to be formed. The outcoming of the soul is a fundamental axis in the Raramuri thought by which the status of the health-illness and lifedeath is explained. The following ethnographic text pretends to contribute to the anthropological knowledge about the cultures in northern, particularly north-western, Mexico.
\end{abstract}

Key words: Ráramuri, person, body, soul 


\section{INTRODUCCIÓN}

La Sierra Tarahumara, región que cubre 17 de los 67 municipios del estado de Chihuahua, posee una extensión de 60,000 kilómetros cuadrados. Actualmente es habitada por cinco grupos humanos: rarámuri, ódame — tepehuanos—, warijó —warijíos—, o’oba — pimas bajos- y mestizos, que aproximadamente suman 90,000 habitantes (Sariego 2002). El poblado Norogachi, donde se realizó esta investigación, ${ }^{1}$ forma parte del municipio Guachochi. Localizado a $27^{\circ} 16^{\prime}$ latitud norte, $107^{\circ} 08^{\prime}$ longitud oeste y a $2,140 \mathrm{msnm}$ en las márgenes del arroyo Peñascos Blancos que afluye al río Uríke. Norogachi fue fundado entre 1640 y 1650 por misioneros jesuitas (Gómez González 1948: 95).

La variabilidad cultural presente al interior de este grupo puede ser explicada en gran medida por: a) el proceso histórico que ha vivido cada región e incluso cada comunidad y b) la relación actual con el estado mexicano y con la población no rarámuri, c) el medio ambiente. Dicha variabilidad ha sido registrada por otros autores en el ritual (Merrill 1992), la lengua (Valiñas 2001) y el vestido (Aguilera 2006), entre ellos. Esto es importante en tanto la información presentada de la persona rarámuri en este texto es una versión, es decir, una actualización de un conocimiento más amplio en transformación permanente; el cual unifica estructuras de pensamiento de larga duración y coyunturas históricas que particularizan el conocimiento. 


\section{LA CONFORMACIÓN DE LA PERSONA}

Los componentes primigenios de la persona rarámuri son el cuerpo, repokára, y el alma, alewá. La unión de ambos origina la vida humana, misma que se gesta en el vientre materno. María Luisa Bustillos en 2004 narró este suceso de la siguiente manera:

\footnotetext{
Cuando una mujer tiene relaciones con un hombre, el hombre penetra su pene en la parte íntima de la mujer, y lo más importante, le regala la mitad de la vida humana, como un esperma, que será el que llegue a unirse con la otra mitad que va buscando el óvulo. Estos dos al encontrase juntos adentro de la mujer, baja el Dios de la vida, Onorúame, para juntarlos aun más y soplarle el alma adentro, para que pueda formarse un ser humano. Una vez que Onorúame baja a juntar un esperma y un óvulo de la mujer, envuelto en una cobija que será la placenta.
}

En esta breve descripción pueden identificarse algunos términos de la medicina alópata que sirven para personificar "la mitad de la vida" —el espermatozoide y el óvulo—. No es necesario hablar de un conocimiento tradicional y uno moderno porque distintas lógicas han conformado un pensamiento actual sobre la conformación de la vida. El conocimiento que los rarámuri manifiestan es uno y sigue una lógica cultural propia. Es por ello que el esperma y el óvulo unidos recibirán de Dios primero el alma en forma de soplo y luego una cobija o placenta que lo cubrirá. 


\section{EL CUERPO}

En Norogachi, el cuerpo es llamado repokára. La palabra "repokára" designa la parte central de la espalda, incluye el pecho y en ocasiones se extiende a todo el tronco. La relación metonímica por la cual esta palabra designa todo el cuerpo deriva de la importancia de dicha zona. Este segmento corporal contiene el corazón, surára, y los pulmones, sonorá.

Dentro de la clasificación rarámuri, el corazón, los pulmones y la tráquea, iwiára; conforman una unidad semántica que explica el desarrollo y el mantenimiento de la vida. La importancia de estos tres elementos radica en las relaciones que mantienen con el alma. El alma habita en el corazón desde el momento de la fecundación, este será su centro anímico por excelencia. Por medio de la sangre que corre por las venas, lawála, la fuerza vital se transmite a todo el cuerpo. La sangre, compuesta del agua y los caldos comestibles consumidos cotidianamente, se forma en el corazón y se filtra en los pulmones. Por ello una expresión de la fuerza vital será el aliento y la respiración. ${ }^{2}$

El profundo vínculo entre el alma y la respiración se expresa en la forma en la que Dios dota de alma a los nuevos seres con su aliento divino. Algunas técnicas de curación también se basan en este principio: soplar en la boca de un enfermo es transmitir la fuerza del alma. Otra manifestación de la noción rarámuri de la vida donde podemos identificar la importancia de estos órganos son los rituales en los que se efectúan sacrificios animales. ${ }^{3}$ 
No todos los órganos del cuerpo tienen la misma importancia. Ya hemos indicado que el corazón, los pulmones y la tráquea — junto con el alma - son parte de una unidad semántica manifestada en la respiración y el aliento. Otro órgano importante para la conformación de la persona es la placenta. Esta es un componente fundamental para la vida futura de un recién nacido, ya que el destino de su primera envoltura determinará su sino. El cuidado inicial de la placenta definirá si el recién nacido logra integrarse a las normas sociales o convertirse en un lowíame, que los rarámuri traducen como "loco", pero que hace referencia más que a una enfermedad física a un tipo de desadaptación social.

Actualmente, algunas mujeres rarámuri utilizan los servicios de salud de gobierno o servicios privados durante el parto. Esto puede tener cierta repercusión en el carácter de sus hijos porque en las clínicas la placenta puede no ser regresada al paciente, en tanto que se concibe como un probable foco de infección. Esto explica, ante los ojos de los mayores, por qué algunos de los jóvenes rarámuri tienen comportamientos negativos frente a las normas sociales.

Una de las polaridades más importantes para comprender el cuerpo humano desde la perspectiva rarámuri es dentro-fuera. Merrill (1987) señala que la noción del dentro y del fuera atraviesa toda la visión rarámuri. Bonfiglioli (2004, comunicación personal) amplía esta idea al afirmar que lo de afuera es potencialmente peligroso pero necesario. Esta polaridad explica la relación del cuerpo con las entidades que lo animan, a través de las salidas del alma. 
EL ALMA

El alma es etérea, como un aire que viaja y dota de fuerza vital al ser. Después de la muerte, las cualidades benéficas de esta entidad se invierten, no así el resto de sus características. Los muertos son como el viento, por ello pueden volar y viajar con más facilidad por los lugares que conoció en vida. El alma — de los vivos y de los muertos— es idéntica a la persona, pero es, como dicen los rarámuri, más ligera. Sin embargo posee la capacidad de sentir, percibir y sufrir daños "físicos" como el cuerpo, compartiendo de esta manera caracteres.

El alma humana tiene la capacidad de entrar y salir del cuerpo. Entre ésta y el cuerpo encontramos una relación de dependencia, la unión de ambas es condición necesaria para la vida. Las salidas del alma pueden ser temporales y parciales —en tanto que solo sale una parte de ella o una de estas entidades por un tiempo limitado — o total y permanente —al salir la totalidad de la entidad para siempre - y se explicarán de acuerdo con la noción que se tenga de esta entidad.

La variabilidad sobre la concepción de almas entre los rarámuri ha sido documentada por Merrill (1992). En Norogachi se concibe la existencia de solo un alma que se fragmenta en un segmento fuerte y uno débil. La variabilidad de almas en número y forma ha sido sustituida por la idea de un alma única. Esto, como advertimos al inicio del texto, nos habla de un conocimiento en transformación. La región de Norogachi se ha caracterizado por una fuerte presencia misionera, lo cual ha influido de manera evidente en sus nociones 
corporales y anímicas. Sin embargo, la redefinición de la cultura se basa en una estructura preexistente, en la cual es fundamental la salida del alma. Así, a pesar de ser un alma unificada tiene la capacidad de salir del cuerpo fragmentándose.

Las salidas del alma

La permanencia del alma o su salida explica la vida, la salud, la enfermedad y la muerte. De esta manera, la unión equilibrada del cuerpo y de las entidades que lo animan será una condición para mantener la vida. Por el contrario, su separación prolongada conducirá a la enfermedad y de ahí a la muerte. Las salidas del alma pueden efectuarse durante la vigilia y el sueño.

En la vigilia, las almas pueden salir por estar en contacto con algunos espacios desconocidos y/o acuáticos- y seres determinados — los witáriki o seres del agua y los muertos - provocando el susto. Aún es preciso realizar una investigación exhaustiva del susto entre los rarámuri, sin embargo, este estado es causa de la salida del alma.

El sueño, rimúma, ${ }^{4}$ puede ser definido como el estado en el que el alma sale del cuerpo mientras la persona duerme, o como las actividades del alma durante este estado. Merrill habla del sueño como "las actividades de una o más de las almas de una persona mientras la persona está dormida, los rarámuri dependen de los sueños para proporcionarles acceso a información y a seres como Dios, están comprometidos con la realidad de los sueños" (1992: 164). Para los rarámuri de Norogachi el sueño o rimúma corresponde a esta 
descripción. Los sueños, según Merrill, son fundamentales para la vida de los rarámuri. A través de ellos explican gran parte de los sucesos de su vida cotidiana y ritual, estos permiten el contacto entre el mundo sagrado y el mundo profano, además de funcionar como el espacio de potencial peligro y curación.

El alma también puede ser dañada en el espacio onírico, puede sufrir males por la acción de diversos seres, entre los cuales predomina la figura del sukurúame o hechicero. Este ser utiliza seres y animales para provocar males. El sukurúame también se vale del pensamiento para ocasionar brujería, sipabúa, ya sea de manera conciente — porque tiene resentimiento hacia alguna persona o por la petición de otras personas- o inconciente. La metáfora más frecuente para explicar la brujería es el acto de comer.

Otros seres que también causan mal en el espacio onírico son los seres del agua, que atrapan las almas para retenerlas en el mundo del agua. El intercambio de comida u otros bienes es recurrente en los sueños y puede tener implicaciones negativas. Esto también se presenta en los sueños donde interactúan los vivos y los muertos. En el primer caso, un alma puede encontrarse con otras de estas entidades de familiares o amigos, que a través de tal dimensión manifiestan un deseo de verse.

En todos los casos presentados, el alma tendenciosamente es raptada, motivo por el cual no es posible encontrarse con el alma de los enfermos en los sueños, ya que están capturadas por alguno de los seres mencionados. Los owirúames o curanderos — los que curan a través 
de los sueños- son las únicas personas que pueden tener acceso a estos espacios para rescatar las almas y llevarlas de regreso al cuerpo.

\section{EMOCIONES Y PENSAMIENTO}

Las emociones conforman la persona rarámuri. Son producto de las relaciones establecidas entre el cuerpo y el alma. En la lengua rarámuri no existe una palabra que resuma conceptualmente las emociones como una totalidad, por el contrario encontramos términos que remiten a diversos sentimientos. Durante el trabajo de campo se han identificado dos emociones que articulan la vida social y emocional de las personas y que conforman un bimonio: Omona y raisa; palabras traducidas al español por los rarámuri entrevistados como "tristeza y alegría" remiten a campos semánticos más amplios.

Ambas emociones son producto y consecuencia de la relación entre cuerpo y alma. Si estas entidades mantienen un equilibrio las personas estarán raisa o alegres, en caso contrario, el desequilibrio llevará a omona o a la tristeza. Por lo tanto, la salud y la enfermedad, así como la vida y la muerte, también estarán resumidas en estas emociones. Este vínculo es explicado a partir del corazón, órgano donde reside el alma y origen de los estados anímicos.

Si una persona está alegre entonces tendrá salud y por tanto vida, tendrá fuerza en el corazón y aliento para seguir caminando. Como vimos a través de la alquimia corporal, corazón-aliento-fuerza corresponden a un mismo campo semántico que remite a la vida. En 
caso opuesto, si una persona está triste, entonces estará enferma y probablemente morirá, estará descorazonada y no tendrá fuerza para seguir caminando. Desde la perspectiva rarámuri, caminar en un contínuum de la vida sobre la tierra y del devenir del cosmos en general (Guillén y Martínez en prensa).

En los sermones se expresa públicamente un discurso sobre las emociones, el cual refleja y perpetúa un estatus quo del universo. Merrill registró el siguiente sermón en Rejogochi el 11 de febrero de 1979: "Regresen contentos a sus casas a lo largo de los caminos de Nuestro Padre y Nuestra Madre. ¿Acaso Los Que Caminan Arriba cuidándonos vacilan en su viaje? Por eso, ustedes deben ser fuertes y estar contentos" (1992: 111). Dicho discurso exhorta a los rarámuri a mantener un estado equilibrado de sus emociones y por tanto de la relación entre el cuerpo y el alma para continuar caminando, esto es, viviendo.

Detenerse implica morir, estar triste conduce a la muerte que más allá de una muerte individual podría llevar a una muerte social e incluso cósmica. El significado de la palabra siwéma puede ilustrarnos en este sentido: “Significa ‘¡no te rajes!'. ‘ No te vuelvas atrás!'. ‘No pierdas tu entusiasmo!’ ‘¡No te descorazones!', y en un sentido más general, ‘¡no estés triste!"” (Merrill 1992: 148). Existen otros dos términos para referirse a la tristeza: ni’wíma y o’mónama, lo que nos interesa subrayar es que los tres son verbos y antónimos del término iwéra, que refiere a la respiración, a la fuerza y a la vida. 
Hasta el momento hemos podido identificar dos campos semánticos que giran alrededor de las emociones que remiten a la alegría y a la tristeza. Por una parte tenemos la alegría vinculada al equilibrio, la salud, la vida, la fuerza, el aliento, el corazón y el caminar — movimiento-; y por otra parte tenemos la tristeza que remite al desequilibrio, la enfermedad, la muerte, la pérdida de fuerza, el descorazonarse y el detenerse — estatismo.

Del corazón también surge el pensamiento, de tal manera que la separación entre pensamiento y emoción se reformula para formar una unidad complementaria. El pensamiento, al igual que las emociones, está valorado socialmente como bueno y malo, positivo y negativo. Si una persona se convierte en un lowiáme tendrá en consecuencia un mal pensamiento que lo llevará a romper las normas sociales, este rarámuri habrá perdido entonces "el buen camino", que es el camino de Dios.

Existen dos figuras en la sociedad rarámuri que son ejemplo del buen pensamiento: los gobernadores, siríame, y los mayoras. Para los rarámuri los gobernadores son los portadores del conocimiento de su cultura. Son responsables de que dicho conocimiento se manifieste en el ámbito ritual y jurídico a través de su actuación —pública y personal— y de los sermones, nawesári. En este sentido, si un gobernador posee conocimiento tendrá que reflejarlo en un buen pensamiento que se manifestará a su vez en buenas acciones; en otras palabras, tendrá que hacer una buena fiesta — conseguir recursos, maíz, organizar a los danzantes, estar pendiente de las necesidades de todos los asistentes- y ser justo en los juicios públicos. 
Si este gobernador posee buen pensamiento también tendrá que reflejarse en su oratoria. Para los rarámuri hablar bien es pensar bien, no existe la formulación hablar mal es igual a pensar bien, o viceversa. Es condición sine qua non poseer un buen pensamiento y una buena palabra. El gobernador está encargado de dar un sermón cada domingo después de misa y durante las fiestas del pueblo. Los "términos para "sermones": nawésare y nátiri [...] comparten la raíz "na-" que quiere decir pensar, pero nawésare y nátiri nombran no pensamientos al azar u opiniones espontáneas sino pensamientos bien elaborados y opiniones cuidadosamente bien consideradas" (Merrill 1992: 102). Cuando un gobernador comienza a actuar mal se dice que piensa mal, por ello no podrá transmitir buenos pensamientos en los sermones.

Esta relación entre el buen pensamiento, el buen habla y la buena acción también se refleja en la figura del mayora. Este es un cargo de carácter moral cuya principal función es aconsejar, por este motivo, deberá ser una persona de buen pensamiento. Aconsejará — dar nawésari o sermón - a los niños que han cometido faltas como robos, golpes, rebeldía, descuidos, etc., y a las personas para unirse en matrimonio. De esta manera

los niños se vuelven buenos pensadores al incorporar los consejos ofrecidos por sus mayores [...] en el caso de los adultos los buenos consejos son necesarios para reforzar la habilidad para pensar bien o para transformar el mal pensamiento de una persona que falla al no actuar de acuerdo con las normas de la conducta apropiada (Merrill 1992: 104). 
La articulación entre pensamiento y acción también está presente en el sueño. Soñar es una acción, y siguiendo a Brambila (1983) uno de los significantes usados para referirse al pensamiento es rimúma, es decir, la capacidad de soñar. Soñar es reflexionar, pensar profundamente, lo que podría estar ligado con el buen pensamiento. Entonces, podemos decir que la tristeza está asociada con el mal pensamiento ya sea propio o provocado por alguien, mientras que la alegría remite al buen pensamiento.

La función social de las emociones y del pensamiento es mantener un equilibrio que permite de alguna manera la convivencia cotidiana y el devenir del cosmos. La expresión de las emociones no solo responde a la valoración positiva y negativa que los rarámuri hacen de las mismas, también al espacio y al tiempo en el que pueden ser expresadas. Existe una normativización sobre el manejo emocional de los individuos que se enseña y aprende a lo largo de la vida.

En primer lugar, el equilibrio emocional manifestado por la alegría debe ser constante en la vida cotidiana. Las emociones, tanto tristeza como alegría, son peligrosas si se intensifican. La exaltación provocada por alguna de estos estados anímicos podría provocar desequilibrios entre el cuerpo y el alma, y entre las personas. En segundo lugar, existen espacios y tiempos determinados para expresar intensamente las emociones. 
Las teswinadas ${ }^{5}$ son el espacio social en el que es permitida la explosión de alegría. La gente bromea y ríe a carcajadas, expresiones poco comunes durante la vida diaria. La alegría está prescrita pues de lo contrario la gente tendería al mal pensamiento y causaría daño a otras personas. A pesar de esta prescripción, las teswinadas también son el espacio donde se expresa el conflicto y otra emoción: el enojo o la rabia, producto del estado de embriaguez, por ello los actos cometidos en dicho estado no serán sancionados.

Cuando una persona está borracha, su alma sale temporalmente del cuerpo y se queda junto a la persona, por ello la gente "no puede pensar bien" y puede cometer actos violentos o actos que rompan las normas sociales. Pero estos actos están justificados, hasta cierto punto, moralmente, dado que la persona no es totalmente dueña de sus acciones, pues nuevamente hay un desequilibrio entre el cuerpo y el alma. Las teswinadas son espacio de infidelidades, robos, asesinatos, golpes, peleas, pero también son espacio de gran diversión. En general hay una distensión de la norma y la inversión se adueña del espacio festivo.

Otro espacio donde se prescribe la alegría es en los rituales mortuorios. El ambiente festivo que predomina en estos momentos responde a la lógica del equilibrio que rige las emociones rarámuri. Las personas sienten tristeza, pero no deberán expresarla, de lo contrario su corazón y su alma se debilitarían, perderían fuerza y aliento. Los muertos, por su parte, desean llevarse consigo a sus parientes para no caminar solos hacia su destino. Si las almas de los deudos están débiles será más fácil que enfermen y mueran. Por ello deben 
expresar alegría, reír y bailar en las fiestas, "estar fuertes" como aconsejan los invitados a los deudos.

La expresión del pensamiento y las emociones rarámuri, como indica Le Breton (1995: 9), está social y culturalmente moldeada. Su manejo responde a una normativización social que permite mantener y reproducir las relaciones entre los sujetos y entre el ser humano y el cosmos. Las emociones y el pensamiento forman parte de la persona, son parte de lo social que construye y moldea a cada ser humano.

\section{CONCLUSIÓN}

Explorar, desde una perspectiva emic, la noción de persona ha mostrado que la oposición cartesiana entre materia y espíritu no es la base del entendimiento rarámuri. Por el contrario, las propiedades del cuerpo y del alma, así como sus interrelaciones, muestran que ambos componentes comparten cualidades, la piel no es el límite del alma, ni el alma lo es del espíritu. La porosidad mutua permite la transmisión de la fuerza vital y el mantenimiento de la vida.

El cuerpo y el alma responden a diversos tipos de clasificaciones, entre ellos de tipo lógico —respecto al espacio y al tiempo—y de tipo social. Bajo esta última podemos enmarcar aquellos factores de la persona rarámuri que surgen de la relación entre cuerpo y alma: el sueño, las emociones y el pensamiento. A través de estos tres elementos podemos confirmar que "la persona es lo que hay de social en nosotros" (Durkheim 2000: 283). Los 
órganos escapan a una lógica de corte biológico y poseen la personalidad misma del sujeto, por ello la placenta está ligada al pensamiento.

Los componentes de la persona, particularmente la fuerza vital contenida en el alma y el cuerpo, vinculan a los sujetos con el orden social y el cosmos. El mundo está poblado por seres ávidos de robar y comer el alma. Por tanto, la salud y la enfermedad, la vida y la muerte, se explicarán a partir del estado del alma en relación con el cuerpo. Las emociones son fundamentales para mantener o provocar dichas situaciones. El factor emotivo también influye en el devenir de las relaciones sociales y del universo en general. Por ello se busca el equilibrio.

Dicho equilibrio resulta ser un principio fundamental en el pensamiento rarámuri. Este debe mantenerse, incluso se podría afirmar que la vida es una lucha por el mantenimiento del equilibrio que en la persona se manifiesta entre sus componentes. De esta forma se debe conservar un equilibrio entre el cuerpo y el alma, un equilibrio en el pensamiento y en las emociones. La pérdida de este principio conduce a consecuencias nefastas que afectan todos los niveles de la existencia.

Aún quedan múltiples espacios por explorar, como el curso de vida y el parentesco, el poder inscrito en el cuerpo y el alma. El proceso para llegar a ser una persona, la tipología de la misma, así como el lugar que cada sujeto ocupa en la retícula social. La ritualidad podría ser un espacio fértil de investigación. Por el momento se puede indicar, que al igual 
Revista Pueblos y Fronteras digital La Noción de Persona en México y Centroamérica
Núm. 4, Dic. 2007 - Mayo 2008

http:// www.pueblosyfronteras.unam.mx

que en otros ejercicios, la noción de la persona rarámuri nos ha mostrado que existen otras formas, culturalmente moldeadas, de comprender y vivir el ser humano. 


\section{BIBLIOGRAFÍA}

Aguilera, Sabina, 2006, "La faja ralámuli, un entramado cosmológico". Tesis de licenciatura. ENAH, México.

Brambila, David, S.J., [1953] 1983, Diccionario Castellano-Rarámuri. Obra Nacional de la Buena Prensa, México.

Durkheim, Émile, [1912] 2000, Las formas elementales de la vida religiosa. Colofón, México.

Gómez González, Jilberto, 1948, Rarámuri. Mi diario Tarahumara. Talleres tipográficos de Excelsior, México.

Guillén, Héctor e Isabel Martínez, en prensa, "Pensamiento y emociones rarámuri, un nuevo campo de indagación". En Memorias del ler. Encuentro de Etnopsicoanálisis y Etnopsiquiatría. ENAH, México.

Le Breton, David, 1995, Antropología del cuerpo y modernidad, Ed. Nueva Visión, Argentina.

Merrill, William L., [1988] 1992, Almas rarámuris. CONACULTA-INI, México.

Merrill, William L., 1987, “The rarámuri stereotype of dreams”. En Dreaming. Anthropological and Psychological Interpretations, editado por Bárbara Tedlock, pp. 194-219. Cambridge University Press, New York.

Sariego Rodríguez, José Luis, 2002, El indigenismo en la tarahumara. Identidad, comunidad, relaciones interétnicas y desarrollo en la Sierra de Chihuahua. INICONACULTA-INAH, México. 
Valiñas, Leopoldo, 2001, "Lengua, dialectos e identidad étnica en la Sierra Tarahumara". En Identidad y cultura en la Sierra Tarahumara, coordinado por Claudia Molinari y Eugeni Porras, pp. 105-126. INAH- Congreso del Gobierno del Estado de Chihuahua, México. 


\section{Notas}

${ }^{1}$ La información de los siguientes apartados fue recolectada en el pueblo de Norogachi y en dos rancherías aledañas: Santa Cruz y Kochérare. El trabajo de campo se llevó a cabo en cuatro periodos: abril de 2002, abril de 2003, octubre 2003 a febrero de 2004 y marzo a abril de 2004.

${ }^{2}$ Merrill (1992: 138) señala la existencia de una relación lingüística entre los términos ariwá e iwigá para hablar de alma y aliento, considerados como uno mismo; esta denominación también se emplea para referirse a la vida en general.

${ }^{3}$ Para los sacrificios animales se emplean vacas y chivos. Se les corta la carótida y la sangre que brota de la herida se ofrece a los cuatro rumbos cardinales. Esta sangre "caliente" es la primera ofrenda para Dios (Onorúame), lo que nos habla de la importancia de esta sustancia.

${ }^{4}$ Merrill advierte que el sueño no es un objeto sino una acción, ya que este es su uso lingüístico: "For the rarámuri, dreams are events, not things; they have no noun that can be glossed as 'a dream', only the verb rimúma, 'to dream'” (1987: 199).

${ }^{5}$ La teswinada es parte de las celebraciones rituales rarámuri en las que se bebe teswino, fermento de maíz. Presenta un carácter colectivo que expresa distintos niveles de relaciones sociales: grupos familiares, rancho, rancherías y poblados. La preparación de "buen teswino" otorga prestigio. Las personas con este reconocimiento serán seleccionadas con mayor frecuencia para elaborarlo por encargo de las autoridades rarámuri o para obtener un cargo ritual. No obstante, el aspecto económico influye en estas decisiones, debe producirse suficiente bebida y comida para todos los invitados. Los momentos y lugares donde se llevan a cabo las teswinadas dependerán del tipo de ceremonia que se realice. Una teswinada puede iniciar a cualquier hora del día, esto dependerá del ciclo ritual y el tipo de festividad. Antes de ser repartida, la bebida ritual debe ser ofrendada a Dios y solo podrá ser distribuida entre los asistentes por el casero o la casera, se entrega en recipientes de cinco a veinte litros. No es bien visto que los asistentes se marchen antes de que se termine de tomar todo el teswino de una casa, que puede ser hasta mil litros. Si una persona ha sido invitada a varias casas deberá asistir a todas ellas, de lo contrario se le reprochará posteriormente su ausencia. Este es el motivo por el cual las borracheras en la teswinada pueden durar días o incluso semanas trasladándose de un lugar a otro. 